\title{
Applications of adaptive variable step-size algorithm in turbulence observation system
}

\author{
Yongfang Wang ${ }^{\mathrm{a}, \mathrm{b}, *}$, Chengdong Yang ${ }^{\mathrm{a}, \mathrm{b}, \mathrm{d}}$, Jianlong Qiu ${ }^{\mathrm{c}}$ \\ a School of Informatics, Linyi University, Linyi, Shandong 276005, P. R. China. \\ ${ }^{b}$ LinDa Institute of Shandong Provincial Key Laboratory of Network Based Intelligent Computing, Linyi University, Linyi, \\ Shandong 276005, P. R. China. \\ 'School of Science, Linyi University, Linyi, Shandong 276005, P. R. China. \\ ${ }^{d}$ School of Automation, Southeast University, Nanjing, Jiangsu 210096, P. R. China.
}

\begin{abstract}
In turbulence observation system, noise signal is random and difficult to identify, which will pollute the real signal and affect the quality of the data. To eliminate the noise signal, the article puts forward a kind of adaptive variable step-size de-noising algorithm. Firstly, raw data is changed into corresponding physical parameters, and spectral analysis is used to analyze the relationship among these parameters, and then, according to the correlation to construct the variable step-size de-noising algorithm, and through error to adjust shape of the step size factor to control the optimal weight coefficient. Finally, simulation and observation data is used to verify the effectiveness of the algorithm, and Goodman's filter algorithm is compared with the algorithm. The results show that the algorithm has higher precision and the noise is effectively reduced. (c)2016 All rights reserved.

Keywords: Variable step-size, spectral analysis, adaptive noise canceller, turbulence observation system.

2010 MSC: 68Q25, 68Q32, 68W40.
\end{abstract}

\section{Introduction}

The research of marine turbulent mixing is a hot spot in marine science, while the turbulence raw data is the basic to study the turbulent motion mechanism, and at the same time, ocean turbulence

*Corresponding author

Email address: greenworld6@163.com (Yongfang Wang) 
observation instrument is the important means of access to obtain ocean turbulence data, it is a highend marine scientific observation instrument. Due to the complexity of ocean internal environment, the turbulence data will be polluted by all kinds of noise signal when instruments observe turbulence, and the noise signal is mainly divided into the following kinds [9]:

(1) The vibration produced by observation platform, flow resistance and circuit noise;

(2) Ocean noise, such as the nonlinear interaction between the opposite direction of the surface waves will produce $5-10 \mathrm{~Hz}$ environment noise;

(3) Man-made noise, such as navigation, industry, and drilling noise;

(4) Biological, seismic noise and rain noise, etc.

The noise includes high frequency and low frequency, which seriously reduces the quality of the data. In recent years, in order to eliminate the noise pollution in turbulence observation, many algorithms are proposed at home and abroad; see, e.g., Lan et al. [7] and $\mathrm{Xu}$ et al. [14] analyzed the noise sources, and through improving the placed way of sensor and add partition to improve the measurement precision, but it cannot effectively eliminate the noise produced in the process of signal transmission. Wilfong et al. [13] used median filter to eliminate noise through statistical analysis of the observation spectrum, and the results are simulated by Monte Carlo method, but the median filter only has a good de-noising effect on impulsive noise. Bakunova and Harlim [1] studied the linear autoregressive model in solving the optimal filtering problem of complex turbulent flow system, and the parameter optimization problem in a minimum of 2 order autoregressive model is investigated. To express noise characteristics likelihood function was applied, and then exponential weighted likelihood model was used to eliminate the noise; see [15] for details. In addition, there are wavelet de-noising algorithms [5, 12] and filtering based on harmonic analysis method, etc. However, due to the turbulence is unsteady and transient change, a random mount of energy will be leaked when these algorithms are used, and so the filtering effects are not the optimal.

Based on motion compensation theory [3], this paper proposes an adaptive variable step-size de-noising algorithm. Adaptive algorithm could automatic adjust processing parameters, boundary conditions, and so forth. On the basis of the data characteristics, the algorithm uses the relationship between the ship movements' data and observation data to construct the de-noising algorithm, and through error to adjust the shape of step size factor to control the optimal weight coefficient in order to realize noise reduction. Then simulation and observation data is used to verify the effectiveness of the algorithm, and the turbulence observation instrument [11] based on the submerged buoy system and it is independent research and development by Ocean University of China, which is taken as real turbulence data acquisition platform. The observation data includes turbulence data and sensors data (such as attitude data and conductivity-temperature-depth data) and Goodman's filter algorithm is compared with the algorithm.

\section{Observation Principle of the Turbulence}

Turbulence observation instrument is the main instrument to obtain turbulence data, through testing the ocean turbulence we can obtain the real data of ocean internal turbulence mixing, and internal turbulence mixing plays a key role in the study of marine. The main structure of the turbulence observation instrument is composed by three parts. In the front of the instrument is the shear probe, which detect the turbulence signal; Second part is the instrument cabin, which contains control board, power board and so on; The last part is the tail, its can keep the balance of the instrument. The whole structure is shown in Figure 1. 


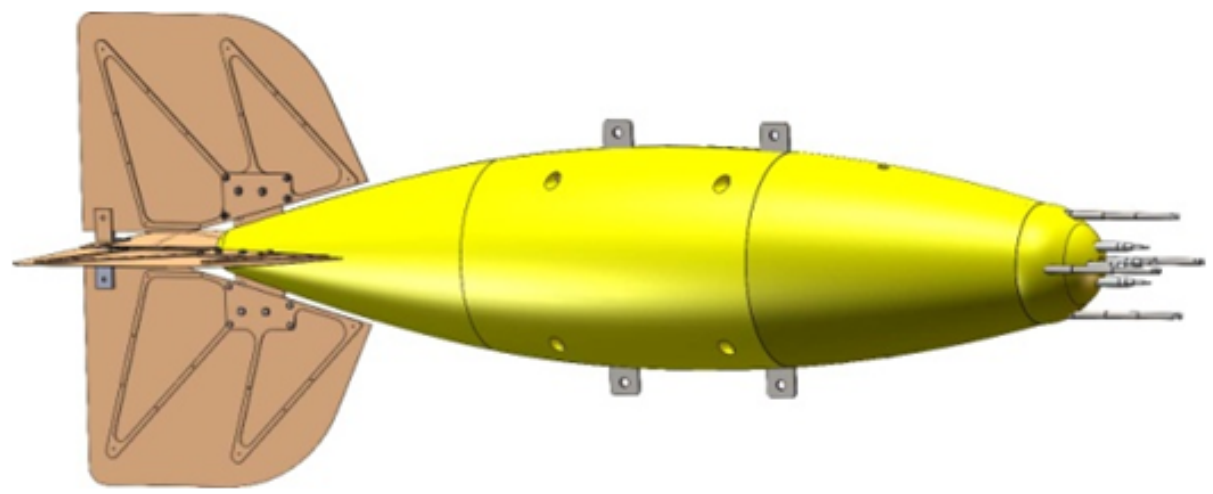

Figure 1: The observation instrument based on the submerged buoy system

In macro-scale, turbulence can be described as irregular random fluctuating of the fluid micelle, and its basic characteristic is the randomness movement of the fluid micelle. Therefore, the data obtained from observation instrument is turbulence fluctuation velocity, and fluctuating velocity is converted to physical parameter to analyze turbulence characteristics. Fluctuating velocity is mainly acquired by shear probe, and the axis of the shear probe is horizontal in fixed anchor turbulence observation platform, so the data is horizontal fluctuating velocity. In the oceanic, due to the shear probe is placed in the front of the observation instrument, it will be easily affected by the vibration of observation instrument. The vibration is random and unpredictable, which is obviously interference the turbulence signal. Soloviev et al. [10] described the vibration phenomenon in the turbulence observation. The principle of shear probe is shown in Figure.2.

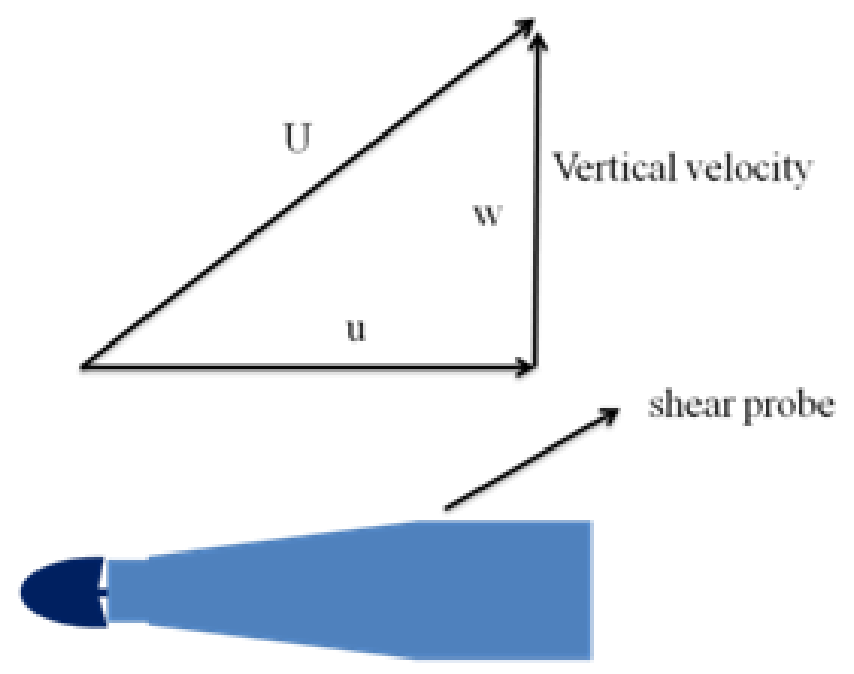

Figure 2: The principle of shear probe

According to Taylor freezing theorem, the time sequence of data got from shear probe is converted into space sequence, and then the horizontal pulsation shear signal is obtained, and the conversion formula is shown as equation (2.1):

$$
\frac{\partial u}{\partial z}=\frac{E^{s}}{\sqrt[2]{2 s w^{2}}}
$$

where $s$ is the sensitivity of the shear probe, $w$ is the velocity along axis of the shear probe, $E^{s}$ is 
voltage value which is got by differential circuit or differential of software tools, $u$ is the speed in the direction of the perpendicular to the axis of the shear probe.

The commonly used method to process turbulent random sampling data is on computer. According to the relevant conditions, it firstly transforms the continuous random signal into discrete digital signal to collect and record, then algorithms of fast Fourier transform (FFT), fast correlation, fast convolution and so on are applied to the electronic computer to convert the signal into quantities, such as turbulence spectrum, the dissipation rate, correlation function and probability density, and finally, the characteristics of the turbulence are analyzed through those quantities. Hence, high quality observation data is the basis to correctly evaluate the turbulence characteristics. Recently, many algorithms [2, 6, 8] are applied to eliminate the noise, but how to accurately eliminate noise interference is still the key point in the turbulence research due to the complexity of the marine environment and the randomness of the noise.

In 2006, Goodman et al. 3] put forward a theory that the observation shear signal $S$ is equal to the shear signal $\widehat{S}$ which has no pollution to vibration signal $V_{0}, V_{0}$ is a parameter that needs to be calculated. Assume that the signal obtained from the shear probe and sensor has a linear relationship between the real environment turbulence signal and vibration signal, then equation (2.2) is got:

$$
\begin{aligned}
S & =\widehat{S}+V_{0}, \\
V_{0} & =B_{i k}^{*}, i=1,2,3, \ldots,
\end{aligned}
$$

where $a_{i}$ is three axises acceleration, $B_{i k}$ is weight coefficient, and $*$ is convolution. The weight coefficient is calculated through Toeplitz matrix and then the noise signal is eliminated from shear signal, which is called motion compensation algorithm.

With the quantity of observation data becomes huge, through matrix to calculating the coefficient is a waste of time, and how to rapidly get effective information from a large amount of data becomes the focus in turbulence research, and noise eliminating is indispensable important point in data processing, which is the basis to get correct data information.

\section{The Adaptive Step-Size Algorithm}

Turbulence is random pulse in three-dimensional irregular, and has the characteristics of nonstationary and transient changes. Therefore, the key problem is to effectively identify and eliminate noise signal in the observation data. In turbulence observation, noise data cannot be observed, but motion of the instrument can be observed and recorded, such as three axises acceleration, which can be used as reference noise signal. In order to verify the correlation between acceleration and shear signal, spectral analysis is used. The result is shown in Figure 3.

The yellow and red vertical dashed lines are shedding frequencies, Dudz is power spectrum density of shear signal, Accx, Accy, and Accz are the power spectrum density of three axises acceleration, they all have peak in the two frequencies, which illustrated that acceleration and shear signal has the same induction of vibration signal. Suppose acceleration signal take as a background noise signal, and the weight coefficient of the noise signal is the key problem to solve.

Adaptive filter [4] is developed in recent years based on wiener filtering, and it is an intelligent de-noising method. The intelligent is mean that the weight coefficient can be adaptive adjustment according to the noise, and achieves the optimal effect of filtering. As it has a stronger adaptability and better filtering performance, it has been widely used in the engineering practice, especially in information processing technology. The research object of adaptive filter is uncertain system or information process. "Uncertainty" here refers to the mathematical model and environment, which 


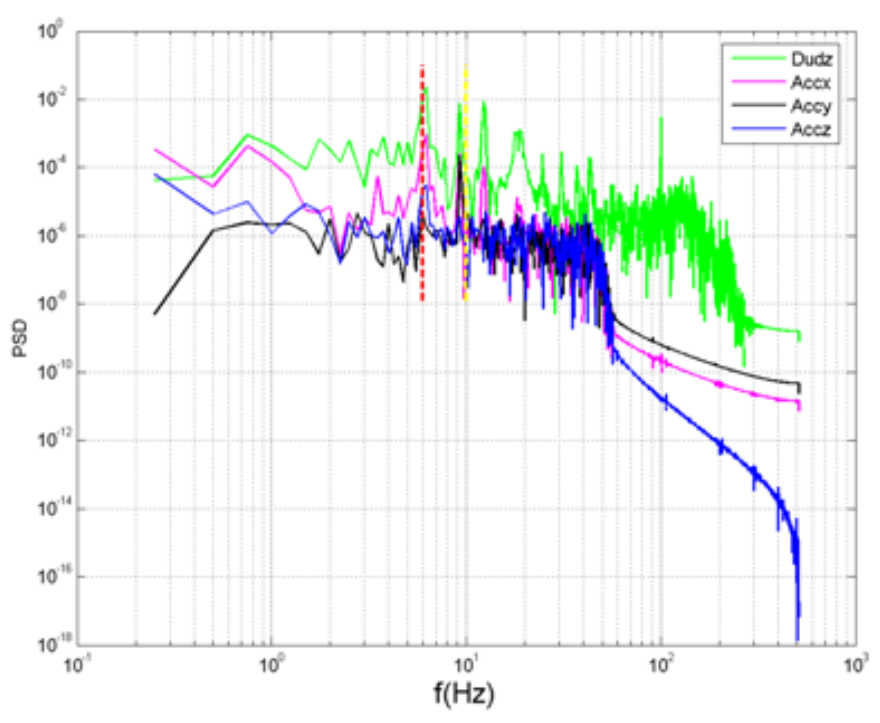

Figure 3: Spectral analysis

contains some unknown factors and random factors. It was completely in conformity with the uncertainty environment of ocean turbulence observation and noise produced by random, which will be used in the turbulence data processing.

The accuracy of the optimal weight coefficient $W(n)$ plays an important role in the filter, the adaptive filter adjusts the weight coefficient by minimizing the mean square error $e(n)$, and at the same time, the robustness of algorithm must be considered. The procedure of the adaptive filter is shown in equation 3.1 :

$$
\begin{aligned}
e(n) & =d(n)-X^{T}(n) W(n), \\
W(n+1) & =W(n)+2 \mu(n) e(n) X(n),
\end{aligned}
$$

where $d(n)$ is desired signal, $X(n)$ is input signal, superscript $T$ is transposition, $\mu(n)$ is step factor which controls the algorithm's stability and convergence rate. Since the different step length will affect the convergence of the algorithm, the paper proposes the variable step size adaptive filter. The variable step size is adaptive refers to the best state of the algorithm. Big step size is used to accelerate convergence speed, when the algorithm is close to the best state, and the step size is change into small to reduce the rate of convergence. In order to get the best overall performance, the algorithm can adjust the step size to select the optimum weight coefficient, which can be expressed as equation $(3.2)$ :

$$
\mu_{0}(n+1)=\alpha \mu_{0}(n)+\gamma e^{2}(n), 0<\alpha<1, \gamma>0 .
$$

Here $\mu_{0}(n+1)$ is defined by follows:

$$
\mu_{0}(n+1)= \begin{cases}\mu_{\max }, & \text { if } \mu_{0}(n+1)>\mu_{\max } \\ \mu_{\min }, & \text { if } \mu_{0}(n+1)<\mu_{\min } \\ \mu_{0}(n+1), & \text { otherwise, }\end{cases}
$$

where $\mu_{\max } \leq \frac{2}{3 \lambda_{\max }}, \lambda_{\max }$ is the largest eigenvalue of autocorrelation matrix $X(n), \alpha$ and $\gamma$ are the constants to control the speed and range of $\mu$. A set of observed data is used to verify the change of $\mu$ that influence on the algorithm convergence. Figure 4 shows the change of $W(n)$ when $\mu$ is chosen as a constant (such as algorithm of LMS), and at the same time, Figure 5 shows the every change of the weight coefficient $W(n)$ when $\mu$ is changed with the errors (such as algorithm of VLMS). 


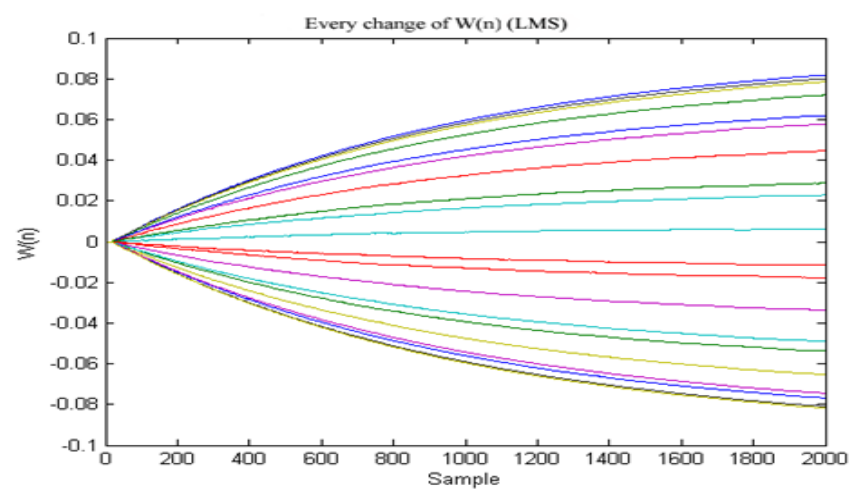

Figure 4: The convergence of $W(n)($ LMS $)$

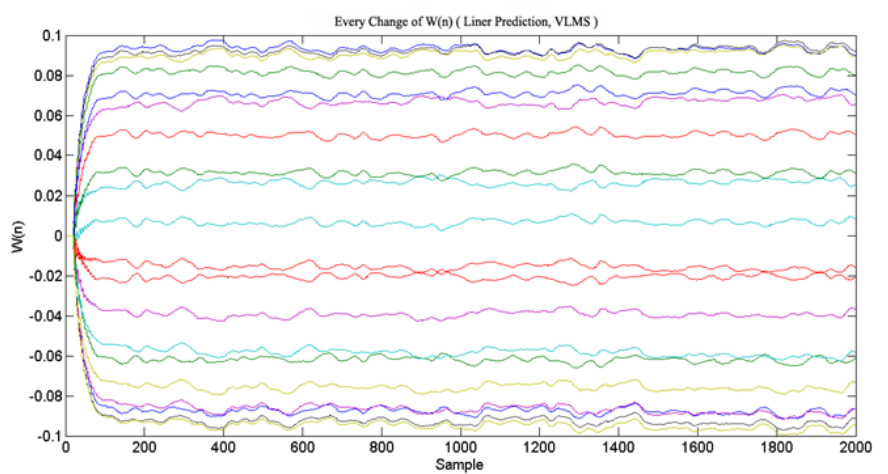

Figure 5: The convergence of $W(n)($ VLMS $)$

Through Figures 4 and 5, we can see that the algorithm has a fast convergence speed when $\mu$ is changed with errors, and equation (3.3) shows that smaller $\mu$ is chosen when the largest eigenvalue of autocorrelation matrix $X(n)$ changed larger, and otherwise the opposite.

\section{Results}

In order to verify the availability of the algorithm, the simulation and South China Sea observation experiment is used. The default length of tap-length is set 20, as the same as Goodman's filter. Define a periodic signal as input signal, and add random noise signal as the original signal, as shown in Figure 6. After using the adaptive step-size algorithm, the cure of the error is shown in Figure 7. Through Figure 7 we can see that the error tends to be stable when the samples are close to 100 . Through Figure 8, 300 samples are used and we can get that the adaptive step-size algorithm is more precision than Goodman's filter by comparing with the desired signal.

Finally, we use the real sea data to validate the validity of the algorithm. The observation data is got from the South China Sea observation experiment (which is carried out by Ocean University of China), the sampling time is 180s, sampling frequency is $1024 \mathrm{~Hz}$, and sampling frequency of acceleration sensor is $120 \mathrm{~Hz}$. The Shear signal (input signal) is shown in Figure 9.

The predict errors are used to verify the algorithm. Figure 10 shows that after using adaptive step-size algorithm (VLMS) and Goodman's filter, the error produced by VLMS is smaller and more concentrated than Goodman's filter, which illustrate that VLMS is more precision than Goodman's filter in turbulence data de-noising. 

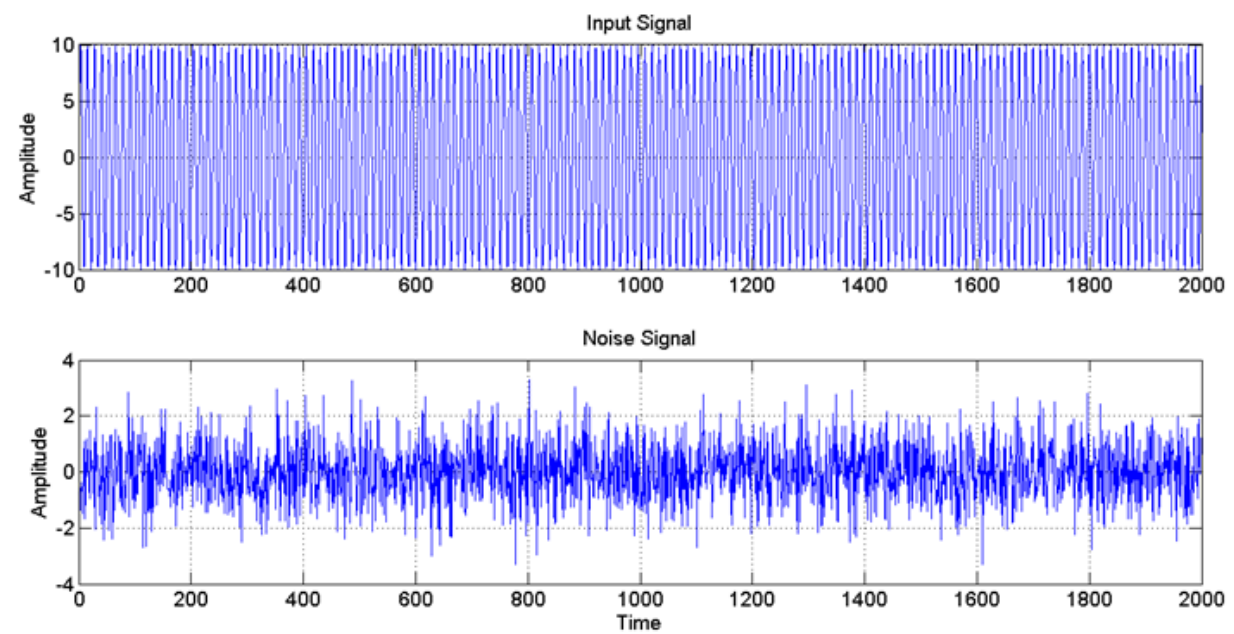

Figure 6: The original signal

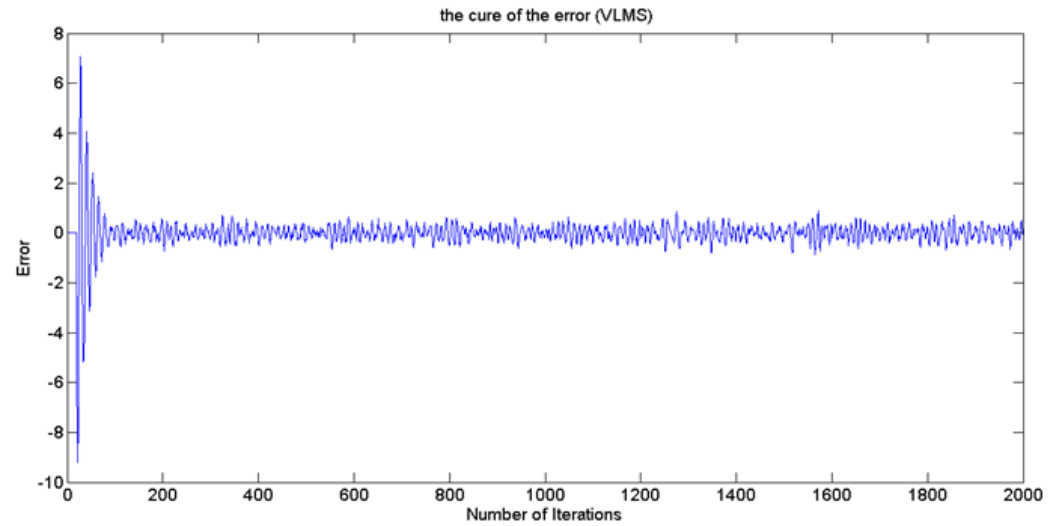

Figure 7: The cure of the error

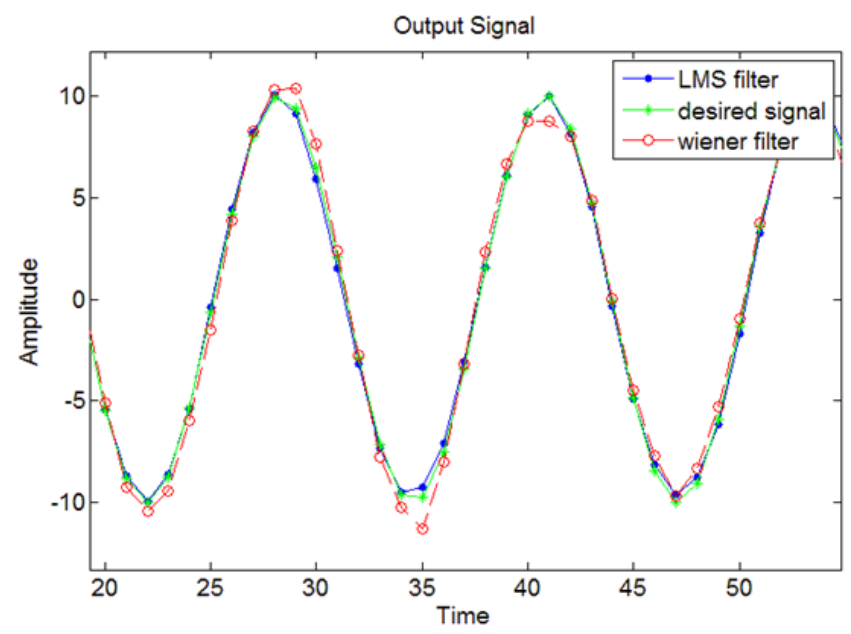

Figure 8: The comparing signal 


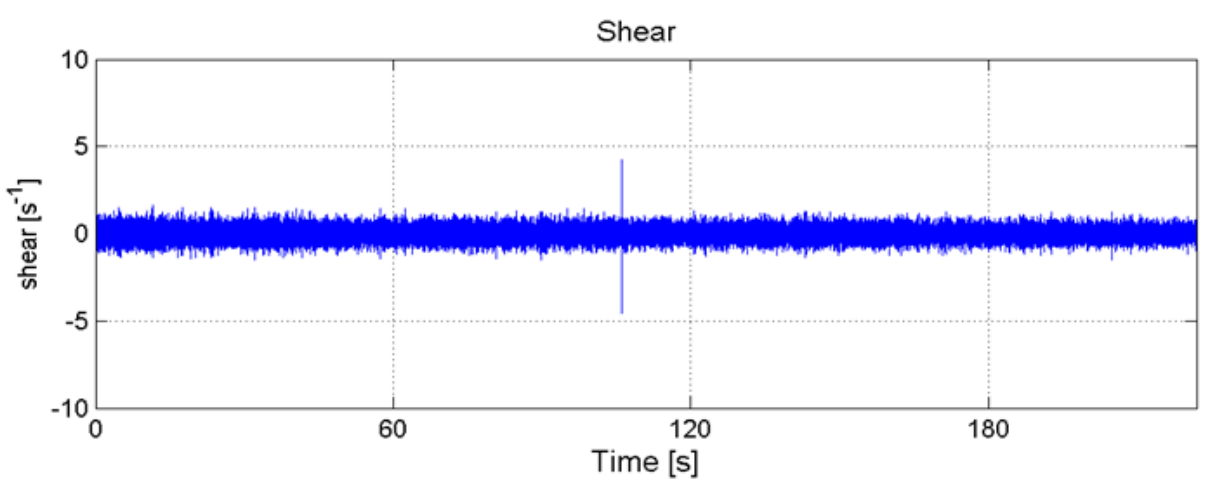

Figure 9: Shear signal

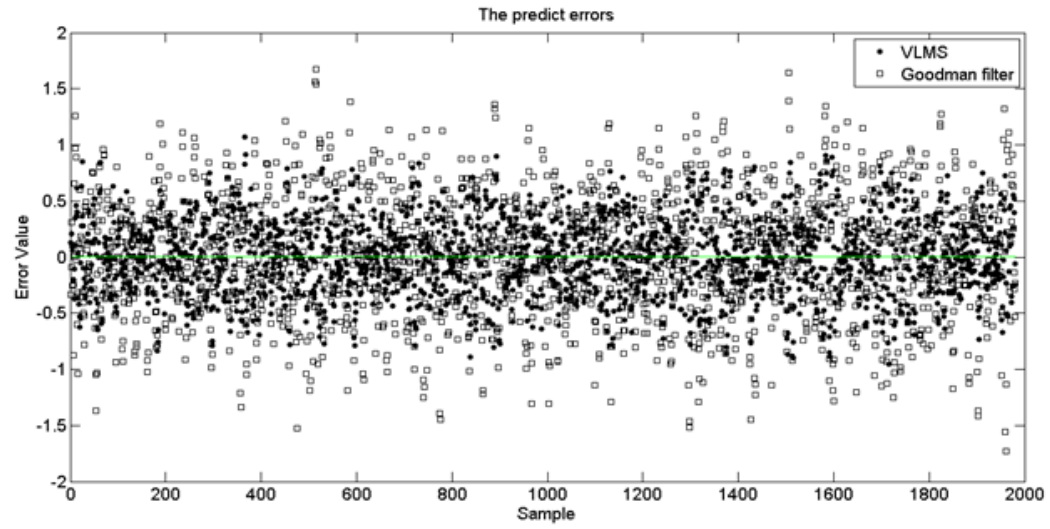

Figure 10: The predict errors

\section{Conclusions}

This paper puts forward a kind of adaptive step-size algorithm in turbulence observation system, and simulation and South China Sea experiment are used to verify the algorithm. By comparing with Goodman's filter, the results show that the algorithm proposed in this paper is more precision and can provide effective data to evaluate the turbulence.

\section{Acknowledgements}

This research is supported by NNSF of P. R. China (Grant Nos. 61503171 and 61403061), NSF of Shandong Province (Grant No. ZR2012FL06), the Doctoral Scientific Research Foundation of Linyi University (Grant Nos. LYDX2015BS001 and LYDX2015BS008), and the AMEP of Linyi University, P. R. China.

\section{References}

[1] E. S. Bakunova, J. Harlim, Optimal filtering of complex turbulent systems with memory depth through consistency constraints, J. Comput. Phys., 237 (2013), 320-343. 1

[2] G. A. Einicke, G. Falco, J. T. Malos, EM Algorithm State Matrix Estimation for Navigation, IEEE Signal Processing Letters, 17 (2010), 437-440. 2

[3] L. Goodman, E. R. Levine, R. G. Lueck, On Measuring the terms of the turbulent kinetic energy budget from an $A U V$, J. Atmospheric Oceanic Tech., 23 (2006), 977-990. 1, 2 
[4] B. Hassibi, A. H. Sayed, T. Kailath, $H^{\propto}$ optimality of the LMS algorithm, IEEE Transactions on Signal Processing, 44 (1996), 267-280. 3

[5] W. A. Kareem, T. Nabil, S. Izawa, Y. Fukunishi, Harmonic analysis filtering techniques for forced and decaying homogeneous isotropic turbulence, Comput. Math. Appl., 65 (2013), 1059-1085. 1

[6] J. L. Kitchen, J. D. Moore, S. A. Palmer, R. G. Allaby, MCMC-ODPR: Primer design optimization using Markov Chain Monte Carlo sampling, BMC Bioinformatics, 2012 (2012), 10 pages. 2

[7] S. Lan, Y. Liu, Y. Wang, J. Liu, Z. Wang, Vibration source analysis and vibration reduction for a vertical microstructure turbulence profiler, J. Vib. Shock, 31 (2012), 5-9. 1

[8] T. D. Mudge, R. G. Lueck, Digital signal processing to enhance oceanographic observations, J. Atmospheric Oceanic Tech., 11 (1994), 825-836. 2

[9] T. Prestero, Verification of a six-degree of freedom simulation model for the REMUS autonomous underwater vehicle, University of California, Davis, (2001). 1

[10] A. Soloviev, R. Lukas, P. Hacker, A near-surface microstructure sensor system used during TOGA COARE. Part II: turbulence measurements, J. Atmospheric Oceanic Tech., 16 (1999), 1598-1618. 2

[11] D. Song, J. Sun, B. Xue, C. Tian, The fixed-point ocean turbulence observation on submerged buoy, IEEE International Conference on Intelligent Control, Automatic Detection and High-End Equipment (ICADE), (2012), 72-77. 1

[12] S. Wang, X. Xiao, Y. Wang, Z. Wang, B. Chen, Denoising method for shear probe signal based on wavelet thresholding, Transactions of Tianjin University, 18 (2012), 135-140. 1

[13] T. L. Wilfong, E. M. Lau, B. L. Weber, D. A. Merritt, S. A. McLaughlin, Median filter effects on radar wind profiler spectral noise statistics, J. Atmospheric Oceanic Tech., 31 (2014), 2088-2093. 1

[14] J. Xu, K. Tang, X. Zhang, Study on mechanism and reduction of hydro-acoustical noise induced by flow over an open cavity based on numerical simulation, Chinese J. Hydrodyn., 5 (2014), 618-629.(in Chinase). 1

[15] X. Zhong, A. B. Premkumar, W. Wang, Direction of arrival tracking of an underwater acoustic source using particle filtering: Real data experiments, TENCON Spring Conference, IEEE, (2013), 420-424. 1 